Cahiers $d u$ MONDE RUSSE

\section{Cahiers du monde russe}

Russie - Empire russe - Union soviétique et États indépendants

45/3-4 | 2004

Varia

\title{
Paul Gregory, The political economy of Stalinism
}

\section{Alessandro Stanziani}

\section{OpenEdition \\ Journals}

Édition électronique

URL : https://journals.openedition.org/monderusse/4193

DOI : 10.4000/monderusse.4193

ISSN : $1777-5388$

Éditeur

Éditions de l'EHESS

Édition imprimée

Date de publication : 1 juillet 2004

Pagination : 677-679

ISBN : 2-7132-2009-2

ISSN : $1252-6576$

\section{Référence électronique}

Alessandro Stanziani, «Paul Gregory, The political economy of Stalinism », Cahiers du monde russe [En ligne], 45/3-4 | 2004, mis en ligne le 06 juillet 2009, consulté le 03 septembre 2022. URL : http:// journals.openedition.org/monderusse/4193; DOI : https://doi.org/10.4000/monderusse.4193

Ce document a été généré automatiquement le 3 septembre 2022

Tous droits réservés 


\title{
Paul Gregory, The political economy of Stalinism
}

\author{
Alessandro Stanziani
}

\section{RÉFÉRENCE}

Paul GREGORY, The political economy of Stalinism. Cambridge, Cambridge University Press, 2004, IX-308 p.

Voici environ trente ans que Paul Gregory nous offre des travaux à la fois novateurs, précis et exhaustifs sur l'économie russe et soviétique. Après une comparaison entre les voies d'industrialisation dans les économies socialistes et de marché et une estimation quantitative du revenu national russe entre le xixe et le xxe siècle, il s'est livré à une série d'analyses sur le fonctionnement de l'économie soviétique jusqu'à sa restructuration actuelle ${ }^{1}$. Il cherche maintenant à rendre compte du fonctionnement de l'économie soviétique sur la base des sources d'archives récemment rendues disponibles. La sélection de ces sources constitue en elle-même un indice de la solidité et de l'intelligence de ce travail. L'auteur exploite en effet les Liternye opisi du GARF contenant la documentation du secrétariat du Sovnarkom en matière économique; il a également consulté les archives des ministères industriels, celles des commissions d'inspection du parti communiste (sur les marchés noirs, les échanges informels, la corruption, etc.); enfin celles du ministère de l'Intérieur et de sa police, tout comme celles du Comité central. Ces multiples fonds ont permis à l'auteur de reconstruire les différents niveaux décisionnels et exécutifs de l'appareil économique soviétique, du Politbjuro au Comité central et au Sovnarkom, aux divers ministères, jusqu'aux glavki, aux entreprises et à leurs responsables.

Le premier chapitre rappelle la bibliographie sur le sujet et précise la méthode de l'auteur. Gregory évoque ainsi les thèses de Hayek et Mises selon lesquelles le plan n'aurait pas été en mesure d'offrir des calculs aussi précis et fiables que le marché (d'où la faillite inévitable de l'expérience soviétique). Il oppose cet argument à celui qui met 
moins en cause le plan que la manière dont il a été mis en œuvre en URSS. L'ambition de l'auteur est précisément d'aller au-delà du modèle théorique de Hayes et Mises ou des partisans de la planification, et d'expliquer la durée, les succès et l'effondrement de l'économie soviétique sur la base de son fonctionnement réel. À cette fin, il interroge les archives à partir de modèles interprétatifs différents: le planificateur soviétique agissait-il comme un savant rationnel, comme un dictateur soucieux de maximiser le revenu ou son pouvoir, ou finalement désireux de maintenir ce pouvoir en conciliant les intérêts des différents lobbies?

Afin de répondre, Gregory étudie la mise en place et le fonctionnement du système soviétique à partir de la révolution et jusqu'à sa chute. Les deux premiers chapitres, qui portent sur les années 1918-1930, sont peut-être les moins novateurs. L'auteur se borne à reprendre les thèses dominantes dans l'historiographie et ses propres travaux précédents. Il montre que la décision de collectiviser l'agriculture fut prise aux plus hauts niveaux du pouvoir et trouva son fondement dans la théorie de l'accumulation primitive de Preobraženskij. Le troisième chapitre complète cette analyse en montrant la mise en place de la hiérarchie politico-économique stalinienne. C'est peut-être le chapitre le plus faible, dans la mesure où il cherche à donner des interprétations en termes de "governance», en ne tenant que faiblement compte des enjeux strictement politiques. En réalité, l'analyse ne devient réellement intéressante qu'à partir du quatrième chapitre où l'auteur identifie les politiques et les conceptions des principaux membres du Politbjuro en matière d'investissement, de salaires et de croissance. Stalin et ses principaux collaborateurs estimaient que l'effort de travail se réduisait en présence d'un salaire soit excessivement bas, soit au-dessus du niveau considéré comme correct (fair) par les ouvriers. Gregory trouve ainsi un fondement rationnel aux enquêtes sur le nastroenie des ouvriers : aux yeux du pouvoir, il s'agissait de connaitre leur sentiment en matière de politiques économiques et de niveau de vie afin de décider comment répartir le revenu entre salaires et investissements. Ce qui explique l'alternance entre brusque accélération de l'investissement et sa réduction pendant les années 1930. Dans ce contexte, comment le plan était-il fixé?

Aux yeux de l'auteur, le plan et ses objectifs constituaient avant tout un instrument de la propagande, d'autant plus que le plan scientifique des économistes fut abandonné dès la fin des années 1920 lorsque les anciens spécialistes prérévolutionnaires furent liquidés, les nouveaux économistes bolcheviks étant censés jouer un rôle purement consultatif. Concrètement, le "plan» était établi sur la base de négociations dans le cadre de la hiérarchie au pouvoir, à savoir: Stalin et le Politbjuro, le Sovnarkom, les ministères "économiques», les glavki, les entreprises. Les niveaux hiérarchiques supérieurs pouvaient certes donner des ordres; cependant, afin d'exercer une autorité réelle et pas seulement formelle, ils étaient obligés de déléguer une partie de l'autorité à leurs subordonnés. Ces derniers étaient même implicitement encouragés à développer des relations informelles entre eux afin de faire face aux directives de leurs supérieurs hiérarchiques. Les relations économiques soviétiques s'appuyaient donc largement sur des contrats implicites. Une certaine indétermination des objectifs et des résultats - et des informations les concernant - était essentielle au fonctionnement du système dans la mesure où elle permettait d'écarter le risque de sanction au cas où l'objectif ne serait pas atteint ou son niveau prévu aurait été jugé insuffisant. Ces comportements se reproduisaient à l'intérieur de chaque institution (ministères, glavki, entreprises, etc.). Dans le même temps, ces relations informelles se développaient seulement en partie dans un sens horizontal, c'est-à-dire entre différents ministères ou glavki. Elles prirent de 
l'ampleur, surtout dans les échanges verticaux, à l'intérieur de la même filière. Le résultat fut une " autarcie progressive » et qui, à terme, finit par miner de l'intérieur le fonctionnement de l'économie soviétique.

La dernière partie de l'ouvrage rend compte d'abord du rôle de la monnaie en URSS, puis de l'effondrement de ce système. Pour ce qui est de la monnaie, l'auteur montre que, contrairement aux déclarations officielles, non seulement elle n'eut pas tendance à disparaître, mais joua au contraire un rôle important dans toute l'histoire de l'économie soviétique. La nationalisation des entreprises et le plan n'empêchèrent pas le recours au crédit et le développement des transactions informelles. Des substituts de la monnaie prirent même de l'essor dès les années 1930.

Sur ces bases, l'auteur explique le déclin, puis l'effondrement de l'économie soviétique, avant tout par la complexité grandissante des marchés et de la bureaucratie économique. L'exigence de spécialisation rendait le "plan» (dans les formes décrites auparavant) encore plus difficile à gérer, alors que l'absence de faillites et de crises empêchait tout renouvellement des entreprises. Gorbačev n'aurait pas compris l'impossibilité de garder une partie de l'économie administrée (propriété étatique) sans avoir recours au binôme coercition-négociation informelle.

En conclusion, The political economy of Stalinism, malgré les réserves évoquées quant à la première partie, constitue un ouvrage indispensable pour comprendre le fonctionnement de l'économie soviétique, qui unit le mérite de l'accessibilité aux non-économistes à la rigueur de l'argumentation.

\section{NOTES}

1. Socialist and non-socialist industrialization patterns, New York, Praeger, 1970 ; Russian national income, 1885-1913, Cambridge, Cambridge University Press, 1983 ; Before command : an economic history of Russia from emancipation to the first Five-Year Plan, Princeton, Princeton University Press, 1994 ; Restructuring the Soviet economic bureaucracy, Cambridge, Cambridge University Press, 1990. 